\title{
Bioinformatics approaches for viral metagenomics in plants using short RNAs: model case of study and application to a Cicer arietinum population
}

\author{
Walter Pirovano ${ }^{1}{ }^{*}$, Laura Miozzi ${ }^{2}$, Marten Boetzer ${ }^{1}$ and Vitantonio Pantaleo ${ }^{3}$ * \\ ' Genome Analysis and Technology Department, BaseClear B. V., Leiden, Netherlands \\ ${ }^{2}$ Institute for Sustainable Plant Protection of National Research Council, Torino, Italy \\ ${ }^{3}$ Institute for Sustainable Plant Protection of National Research Council, Bari Research Unit, Bari, Italy
}

\section{Edited by: \\ Alejandro Reyes, Universidad de los Andes, Colombia}

\section{Reviewed by:}

Carmen Hernandez, Consejo Superior de Investigaciones Científicas, Spain Vicente Pallas, Instituto de Biologia Molecular y Celular de Plantas Polytechnic University of Valencia-Spanish National Research Council, Spain

\section{*Correspondence:}

Walter Pirovano, Genome Analysis and Technology Department, BaseClear B. V., Einsteinweg 5, 2333CC Leiden, Netherlands e-mail: walter.pirovano@baseclear.nl; Vitantonio Pantaleo, Institute for Sustainable Plant Protection of National Research Council, Bari Research Unit, Via Amendola 122/d, 70126 Bari, Italy

e-mail: vitantonio.pantaleo@cnr.it

\begin{abstract}
Over the past years deep sequencing experiments have opened novel doors to reconstruct viral populations in a high-throughput and cost-effective manner. Currently a substantial number of studies have been performed which employ next generation sequencing techniques to either analyze known viruses by means of a reference-guided approach or to discover novel viruses using a de novo-based strategy. Taking advantage of the well-known Cymbidium ringspot virus we have carried out a comparison of different bioinformatics tools to reconstruct the viral genome based on 21-27 nt short (s)RNA sequencing with the aim to identify the most efficient pipeline. The same approach was applied to a population of plants constituting an ancient variety of Cicer arietinum with red seeds. Among the discovered viruses, we describe the presence of a Tobamovirus referring to the Tomato mottle mosaic virus (NC_022230), which was not yet observed on C. arietinum nor revealed in Europe and a viroid referring to Hop stunt viroid (NC_001351.1) never reported in chickpea. Notably, a reference sequence guided approach appeared the most efficient in such kind of investigation. Instead, the de novo assembly reached a non-appreciable coverage although the most prominent viral species could still be identified. Advantages and limitations of viral metagenomics analysis using sRNAs are discussed.
\end{abstract}

Keywords: bioinformatics, chickpea, ancient varieties, plant viruses, reference sequences, de novo assembly

\section{INTRODUCTION}

Over the past years deep sequencing experiments have opened novel doors to reconstruct viral populations in a high-throughput and cost-effective manner (Barba et al., 2014; Massart et al., 2014). Currently a substantial number of studies have been performed which employ next generation sequencing (NGS) techniques to either analyze known plant viruses by means of a reference-guided approach or to discover novel plant viruses using a de novo-based strategy (Kreuze etal., 2009, 2013; Navarro et al., 2009; Szittya et al., 2010; Wu et al., 2010; Giampetruzzi et al., 2012; Loconsole etal., 2012; De Souza etal., 2013; Candresse et al., 2014; Seguin et al., 2014; Marais et al., 2015). Despite of the significant advances made by sequencing technologies only a few methods have been proposed to specifically analyze viral metagenomes, especially if compared to the number of tools designed for, e.g., bacterial metagenome analysis (Schloss et al., 2009; Huson et al., 2011). At least in part this can be attributed to the fact that most viruses are still undiscovered; it has been suggested that at present less than $1 \%$ of the extent of viral diversity has been explored (Mokili et al., 2012). Moreover, viral communities tend to be phylogenetically distant from each other and therefore virus discovery and reconstruction heavily relies on de novo approaches. Another hurdle resides in the fact that viral populations are highly heterozygous which is mainly due to the low fidelity of the viral polymerase. This leads inevitably to a high mutation rate and consequently high variation even within the same populations that comprise a viral quasispecies (Domingo et al., 2012). Assembly tools of short sequence reads such as De Bruijn graph-based methods (Zerbino and Birney, 2008) are in principle designed for linear assembly of less diverse haploid and diploid genomes. As a result the assembly of viral (meta)genomes often leads to a substantial amount of contigs with generally a very short average length. Thus subsequent amplification of the resulting fragments using traditional methods (such as PCR and Sanger sequencing) is often essential to extend the draft assembly.

Also it should be mentioned that the chance of properly reconstructing one or more viral taxonomies heavily depends on the quantity of viral genomes present in the input sample. Given that viruses cannot easily be isolated, generally a high sequencing coverage is necessary to pick up all relevant viral genomic material within a plant sample. Alternatively, virus enrichment is needed (Roossinck, 2012). In other words, projects that aim to characterize viral (meta)genomes in plants can become very costly as these are mostly based on sequencing total DNA or RNA libraries that contain only a small fraction of viral material.

The silencing-based antiviral plant response may help somehow in this deal; it implies the recognition of double-stranded 
(ds) or ds-like RNAs of viral origin by members of plant Dicers (DCLs; Aliyari and Ding, 2009). The recognized viral RNAs are then processed by DCLs into viral small interfering RNAs (v-siRNAs; reviewed by Ding and Voinnet, 2007 and Ruiz-Ferrer and Voinnet, 2009). Two distinct classes of vsiRNAs have been identified: primary v-siRNAs, which result from the DCL mediated cleavage of an initial trigger RNA, and secondary v-siRNAs, which require a plant RNA-directed RNA polymerase (RDR) for their biogenesis (Wassenegger and Krczal, 2006; Donaire et al., 2008; Ruiz-Ferrer and Voinnet, 2009; Vaistij and Jones, 2009; Garcia-Ruiz etal., 2010; Wang et al., 2010). The amplification and high level of v-siRNAs accumulation in many but not all virus infections depends on the combined activity of the host-encoded RDRs such as RDR1, RDR2, and RDR6 with other factors such as the RNA helicase SDE3. The amplification mechanism may result in production of secondary amplified v-siRNAs also in case of weakly induced silencing (i.e., low accumulation of viral RNAs; Garcia et al., 2012).

v-siRNAs can also be successfully used to cover known viral genomes by aligning reads to the reference sequences (ref_seq), thus providing a simple method for detection of known viruses and viroids and their variants (Navarro et al., 2009; Pantaleo et al., 2010). In addition, Kreuze et al. (2009) have used at first sRNA libraries for de novo reconstruction of the complete genome of a known plant RNA virus from multiple contigs of v-siRNAs. Moreover v-siRNAs can be used for non-homologous discovery of novel plant infectious entities (Wu et al., 2012). The deepness and the low level of bias of sRNAs are key factors for the success of either reference alignment and de novo assembly based approaches. Seguin et al. (2014) have demonstrated that is possible to reconstruct the entire genomic master sequence of DNA and RNA viruses from both model and crop plants using v-siRNA libraries when sequencing approximately 20 million deep sRNA libraries. Other research groups have spent efforts to demonstrate that bias in cloning procedures may hide some of the sRNAs and therefore they have studied and developed alternative strategies to reduce such bias (Sorefan et al., 2012).

In the present paper we analyze a specific sRNA library from leaves sampled within plants constituting a Cicer arietinum ancient variety (Red of Ruvo, Apulia-Italy) and from leaves of Nicotiana benthamiana plants infected with the Cymbidium ringspot virus $(C y m R S V)$ in a ratio of approximately 1000 to 1 . The presence of v-siRNAs from CymRSV allows us to compare different bioinformatics tools developed for reference-guided or de novo assembly based approaches. Protocols were also applied to the viral metagenome of $C$. arietinum. We find that a reference-guided approach is very successful in the reconstruction of the most abundant viruses. Instead de novo approaches clearly suffer from the heterogeneity within viral populations. Among the discovered viruses, we describe the presence of a Tobamovirus referring to the Tomato mottle mosaic virus (ToMMV; NC_022230), which was not yet observed on C. arietinum and also not yet revealed in Europe, and one viroid referring to Hop stunt viroid (HSVd; NC_001351.1) never reported in chickpea. Accordingly, we discuss our findings and provide suggestions that aim to discover plant-viruses using a cost-effective approach based on sRNA sequencing.

\section{MATERIALS AND METHODS PLANT MATERIALS, VIRUS, RNA EXTRACTION, AND SMALL RNA SEQUENCING}

The use of wild type $N$. benthamiana plants and infection with CymRSV in vitro transcripts was previously described (Pantaleo etal., 2007; Pantaleo and Burgyan, 2008). The plant growth chamber was set with $10 \mathrm{~h}$ in light and $14 \mathrm{~h}$ in dark at $22^{\circ} \mathrm{C}$. Seed population constituting an ancient variety of C. arietinum named "Red Chickpea of Cassano delle Murgie" accession "Red of Ruvo" (in collection at Mediterranean Germplasm Database, http://ibbr.cnr.it/ibbr/resources/mediterranean-germplasmdatabase) was grown in an open air collection field. Plant leaf material representing the entire population (i.e., one leaf per plant covering the $30 \%$ of the plants) was collected at flowering stage and bulked. Total RNA was extracted from plant tissues using TriReagent (SIGMA) following manual instructions. Low molecular weight RNA was enriched as previously described (Johansen and Carrington, 2001) and mixed in a ratio of 1000 (chickpea) to 1 (N. benthamiana) in amount. Subsequently, libraries of sRNAs were produced using a TruSeq Small RNA Sample Kit (Illumina) and sequenced with standard sequencing oligos on the Illumina HiSeq 2500 platform. Short sequence reads were generated using bcl2fastq software ( $\mathrm{v}$ 1.8.3). The dataset has been deposited in GEO Omnibus under the entry code GSE63378.

\section{BIOINFORMATICS}

Small RNA adapters were removed from the Illumina sequence reads using the "Trim sequences" option of the CLC Genomics Workbench (v 6.0.4). For the ref-seq based approach, the resulting sub-reads were aligned against the CymRSV (NCBI accession code NC_003532) and ToMMV (NCBI accession code NC_022230) reference genomes using the "Map reads to reference" option of the CLC Genomics Workbench (v 6.0.4). For the de novo based approach assemblies were generated, respectively, with Velvet version 1.2.10 (Zerbino and Birney, 2008), Oases version 0.2.08 (Schulz et al., 2012), and MetaVelvet (Namiki et al., 2012). Alignment of the assembled contigs against the CymRSV and ToMMV reference genomes was performed using Burrows-Wheeler Aligner (BWA) v. 0.7.7 (Li and Durbin, 2009). From the alignment consensus sequences were generated using SAMtools version 0.1.19 ( $\mathrm{Li}$ et al., 2009). SNP detection was performed with Nucmer which is part of the MUMmer analysis package (version 3.22; Kurtz et al., 2004). Graphical alignment visualization were generated using the Integrative Genomic Viewer (IGV; Robinson et al., 2011). All software was used with default settings unless otherwise specified in text and figures.

\section{RESULTS}

\section{SHORT RNA DATASET}

sRNAs specifically of 20-27 nucleotides with $5^{\prime}$-phosphate and $3^{\prime}-\mathrm{OH}$ (likely to be DCL products) were isolated from C. arietinum "Red of Ruvo" and from a CymRSV-infected N. benthamiana and further identified by high-throughput Illumina sequencing. The library yielded in total more than 11 million reads (table in Figure 1A) with a minimum and maximum length of 16 and $27 \mathrm{nt}$ (Figure 1B). A consistent fraction of these (approximately 6 million) were $24 \mathrm{nt}$ in length (Figure 1B) and this is in line with 


\section{A}

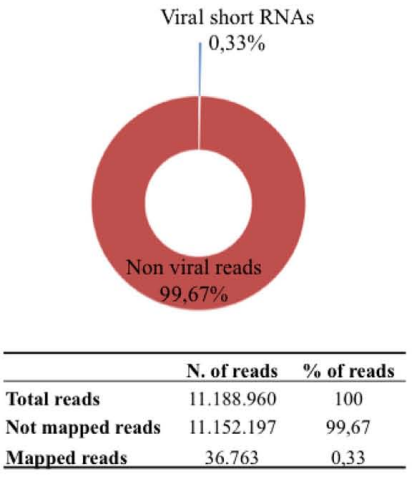

B

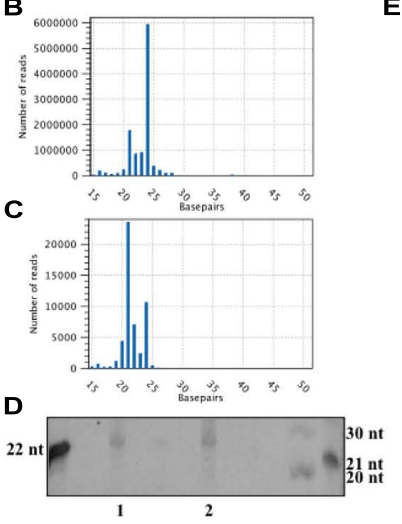

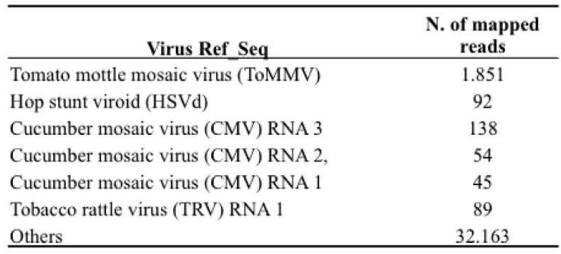

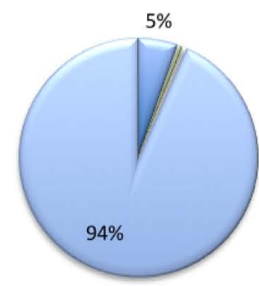

$\square$ ToMMV

$\square$ HSVd

GCMV RNA3

$\triangle$ CMV RNA2

$\square$ CMV RNA1

$\triangle$ TRV RNA1

$\triangle$ Others
FIGURE 1 | Statistics of the short RNA library from Cicer arietinum ancient variety "Red of Ruvo." Pie graph and table resuming the dimension of the library and the fraction of sRNA reads mapping with plant virus ref_seq dataset (A), distribution of sRNA length of non-viral reads (B) and viral reads $(\mathbf{C})$, Ethidium bromide staining of sRNA from $C$. arietinum (1), not infected Nicotiana benthamiana (2; D), table and pie graph resuming viral reference sequence (ref_seq) represented in the sRNA library (E) observations in ethidium bromide staining of the polyacrylamide isolation gel (Figure 1D, lane 1). The abundance of $24 \mathrm{nt}$ sRNAs found in chickpea also agrees with previous studies showing that in plants, except for a few species, the 24 nt sRNAs are more abundant than the 21 nt class (Rajagopalan et al., 2006; Moxon et al., 2008; Pantaleo et al., 2010). Accordingly, Figure 1D shows that the sRNAs from C. arietinum (lane 1) and not infected N. benthamiana (lane 2) equally migrate as they are of the same size.

Those sRNAs flanked by the $3^{\prime}$ and $5^{\prime}$ TrueSeq Illumina adapters were compared with a plant virus reference dataset (ftp://ftp.ncbi.nih.gov/refseq/release/viral/), which is defined by collection of 1.677 unique plant virus master sequences. In total 36.763 reads could be mapped to the plant virus reference dataset (excluding those from CymRSV), thus the v-siRNAs constituted only $0,33 \%$ of the entire library (Figure 1A). More than half of the v-siRNAs were of length $21 \mathrm{nt}$ (ca. 23.000), whereas those of length 22 and $24 \mathrm{nt}$ were less abundant (of ca. 7.000 and 11.000, respectively; Figure 1C). This distribution recapitulates what was previously observed in plant virus infections, particularly in those infected with RNA viruses, and it mirrors the plant DCLs activity involved in RNA-silencing-based antiviral activity (reviewed by Shimura and Pantaleo, 2011). The most represented viral genomes by viral reads comprise the Tobamovirus ToMMV (i.e., 1.851 reads), the Hop stunt viroid (HSVd; i.e., 92 reads), the Cucumber mosaic virus (CMV; i.e., 45, 54, and 138 reads map against CMV RNA1, 2 and 3, respectively) and the RNA 1 of the Tobacco rattle virus (TRV; i.e., 98 reads; Table in Figure 1E). The above mentioned viral reads all together represented about $4.5 \%$ of the entire population of siRNAs that map against the plant virus dataset, indeed most viral reads are scattered in exiguous number across viral ref_seq (i.e., less than 10 unique reads per ref_seq). Moreover, these putative viral siRNAs align to unrelated viruses (i.e., belonging to different viral families) thus not suggesting the need for further investigations.

\section{REFERENCE SEOUENCE-GUIDED ASSEMBLY}

As mentioned above and detailed in the "Materials and Methods" section, the sRNA library under analysis included a small fraction of siRNAs from CymRSV-infected N. benthamiana. Thus, we have first reconstructed the CymRSV genome through alignment of the sRNA reads against its ref_seq (NCBI accession code NC_003532). The alignment statistics are shown in Table 1. A total of 364.590 sRNAs reads, with an average length of $21 \mathrm{nt}$, mapped onto the 4.733 nt long ref_seq. Each nucleotide of CymRSV was covered by sRNA reads 77,03 times on average and all together the reads were able to reconstruct $99 \%$ of the entire genome (the final consensus sequence comprises 4.698 of the original $4.733 \mathrm{nt}$ ). Subsequent variant calling revealed the presence of 13 SNPs; such degree of variability between the consensus sequence and the ref_seq is in agreement with previous findings for Tombusvirus variability at 3 days after inoculation of an in vitro transcript (Russo et al., 1994).

The same approach was used for the reconstruction of ToMMV (NCBI accession code NC_022230). This virus was the best represented by viral reads population in chickpea (Figure 1E). Individual alignment of all reads against exclusively NC_022230 shows that a total of 1.909 sRNAs (with an average length of 21,55 nt) could be mapped (Table 2). Given the reference length

Table 1 | sRNA alignment statistics against the Cymbidium ringspot virus (CymRSV) reference sequence (ref_seq) NC_003532.

Length of the reference CymRSV sequence NC_003532

4.733

Number of mapped reads

364.590

Average length

21,08

Average coverage

77,03

Number of nucleotides in consensus sequence

4.698

Fraction of reference covered

0,99

Number of SNPs with NC_003532 
Table 2 | sRNA alignment statistics against the Tomato mottle mosaic virus (ToMMV) ref_seq NC_022230.

Length of the reference ToMMV sequence NC_022230

6.398

Number of mapped reads

1.909

Average length

21,55

Average coverage

6,4

Number of nucleotides in consensus sequence

5.582

Fraction of reference covered

0,87

Number of SNPs with NC_022230

39

of $6.398 \mathrm{nt}$, each ToMMV nucleotide was represented 6,4 times on average; in total $87 \%$ of the entire genome was covered at least one time (the consensus sequence covered 5.582 out of $6.398 \mathrm{nt}$ ). Finally, the variant calling analysis revealed the presence of 39
SNPs. Notably, the number of SNPs found is sensibly higher than those found in the model system CymRSV. This is particularly interesting if we consider the incidence of SNPs in relation to the total ToMMV v-siRNAs (i.e., 1.909) versus those of CymRSV (364.590). Nonetheless, the large variability encountered in the present metagenomics investigations on field-cultivated plants is fully in line with previous reports for other non-in vitro plant/virus systems (Seguin et al., 2014).

The graphic distribution of mapped reads against CymRSV and $T o M M V$ is reported in (Figures $\mathbf{2 A , B}$ respectively). The graphic representation shows that ToMMV is almost entirely covered by v-siRNAs in a manner that is at least visually similar to that of CymRSV, thus reproducing a high genome coverage of $99 \%(C y m R S V)$ and $87 \%$ (ToMMV) already indicated in Tables 1 and 2. Also a viroid referring to HSVd (NC_001351.1) was almost entirely reconstructed with only 92 reads when applying

A

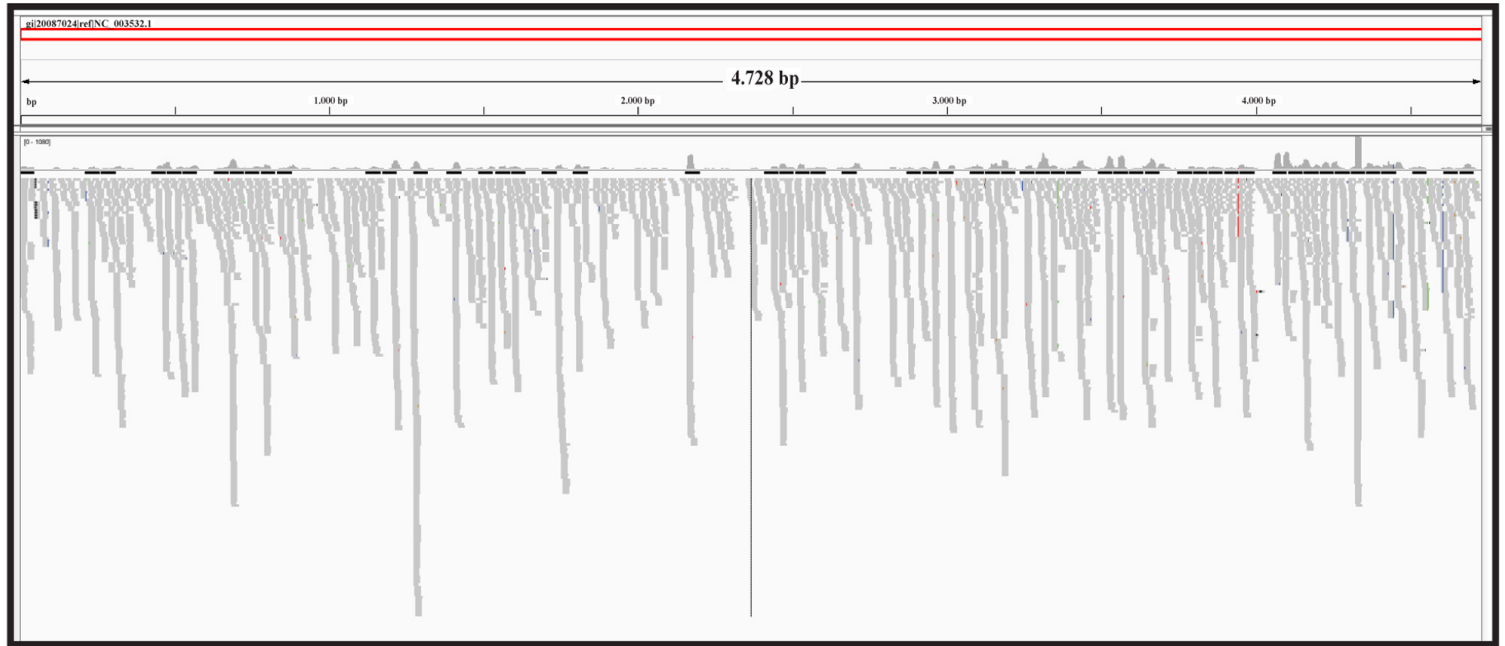

B

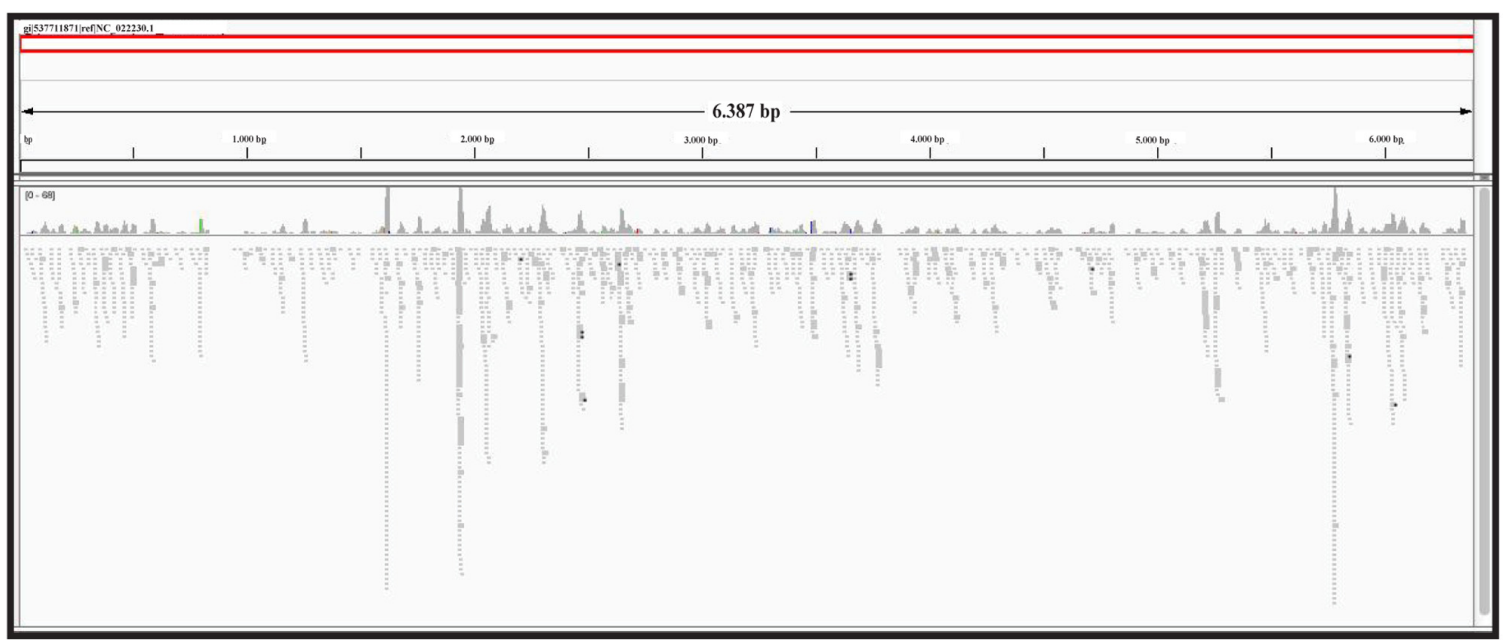

FIGURE 2 | Reference sequence based approach. sRNAs alignment to the CymRSV ref_seq NCBI\# NC_003532 (A) and to ToMMV ref_seq NCBI\# NC_022230 (B). 


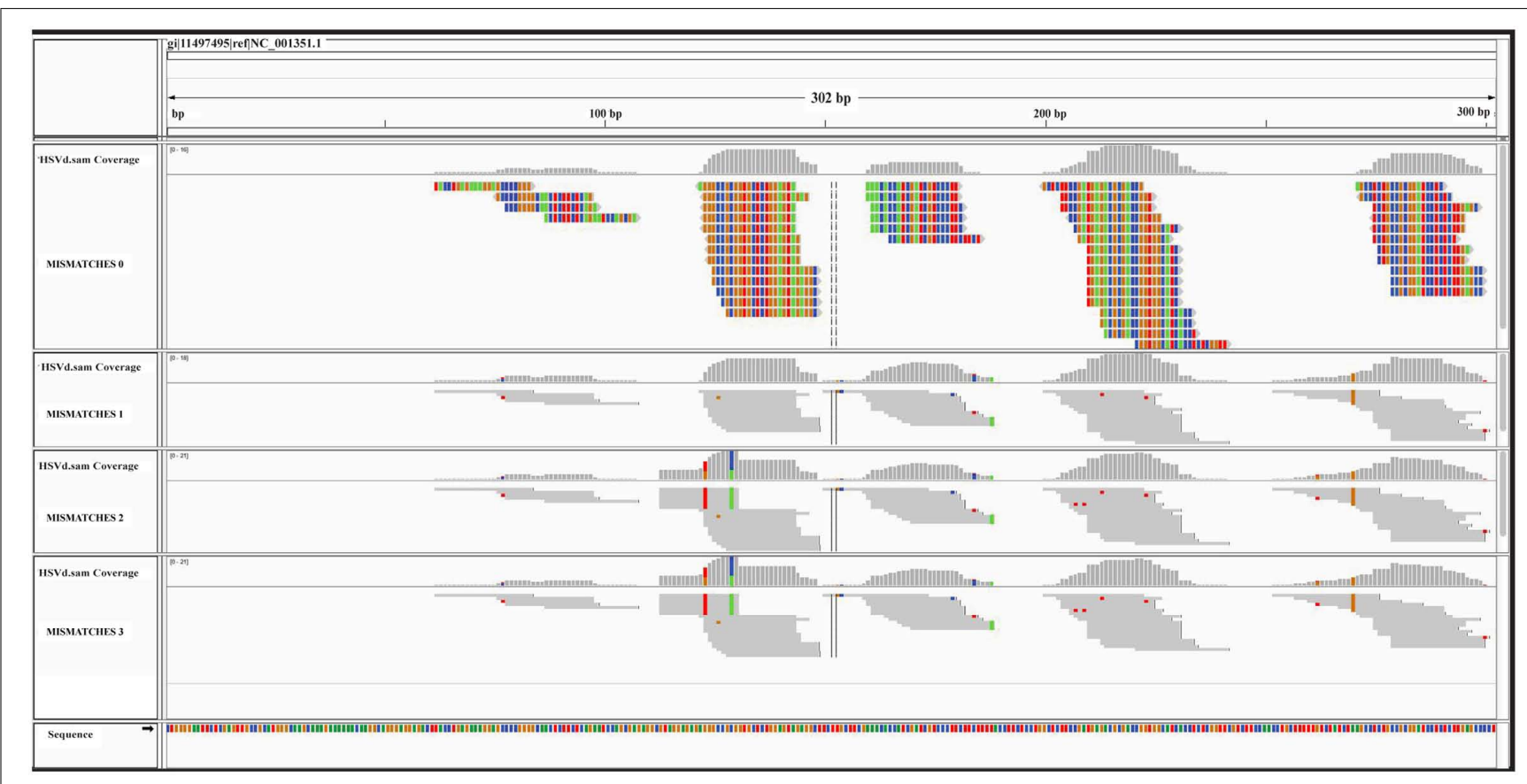

FIGURE 3 | Graphical overview of the ref_seq-based approach. SRNA alignments against the HSVd ref_seq NCBI\# NC_001351 are displayed. The four consecutive panels correspond to $0,1,2$, and 3 mismatches used in
BWA alignment. All nucleotides are colored in the expanded graphic representation (mismatches $=0$ ): $\mathrm{C}$ in blue, $\mathrm{G}$ in brown, $\mathrm{A}$ in a green, and $\mathrm{T}$ in red. Mismatches are colored in not expanded representations. the same settings as for CymRSV and ToMMV (Figure 3). The shortness (302 bases) of the viroid allowed us to check whether a better coverage could be obtained by introducing mismatches in BWA alignment protocol. Indeed, some gaps were covered when using two mismatches (Figure $\mathbf{3}$ ) and at three mismatches no further improvement was obtained. Still, the $5^{\prime}$ part of the viroid (position 1-60 of the released HSVd ref_seq NC_001351), upstream the central conserved domain (CCD; Keese and Symons, 1985; Visvader and Symons, 1985) could not be covered by introducing single variants in the alignment with the ref_seq (see Discussion).

\section{DE NOVO BASED APPROACHES AND v-siRNAs ASSEMBLY}

Given that at present ca. 900 species of plant viruses have been determined (Ninth Report of the International Committee on Taxonomy of Viruses. Elsevier Academic Press, 2012) in most cases no good reference (or master consensus genome) is available. In this scenario de novo assembly of viral genomes should be considered

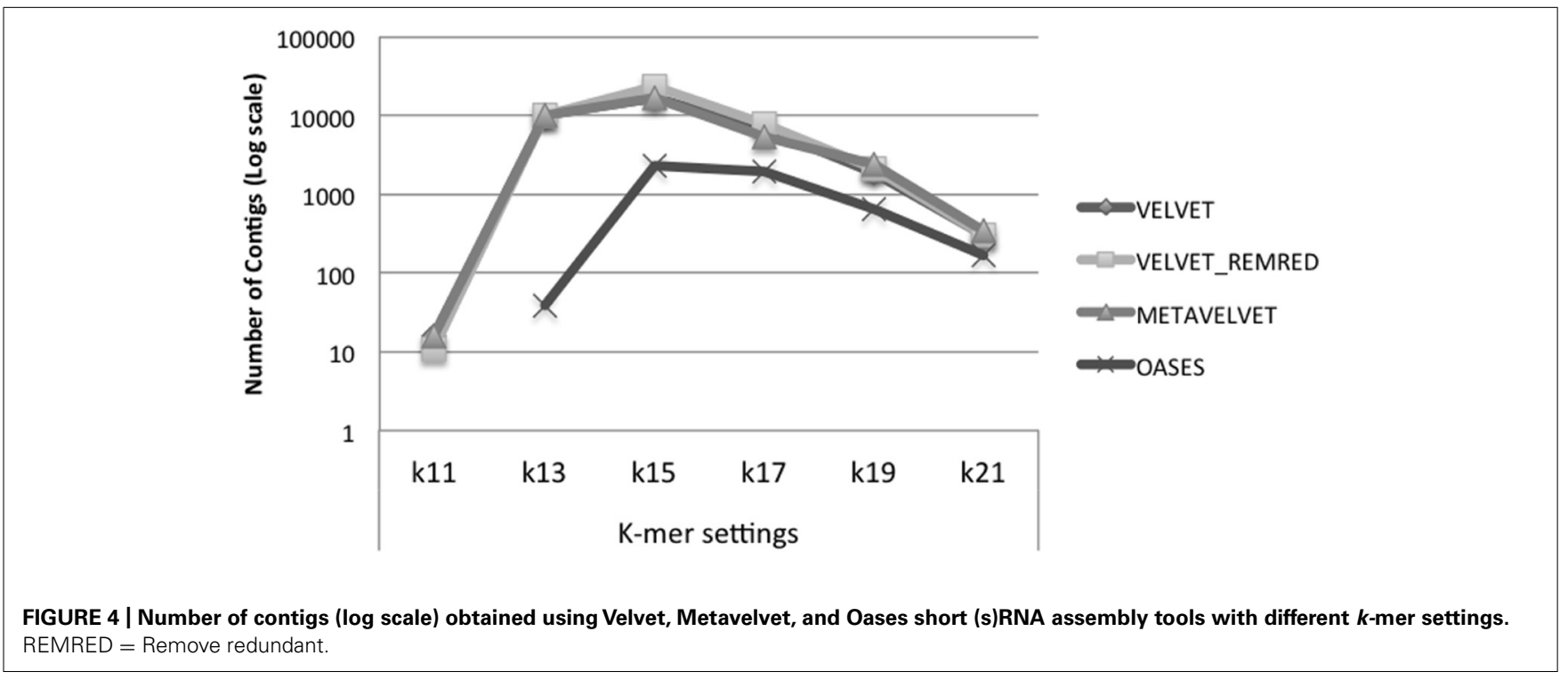




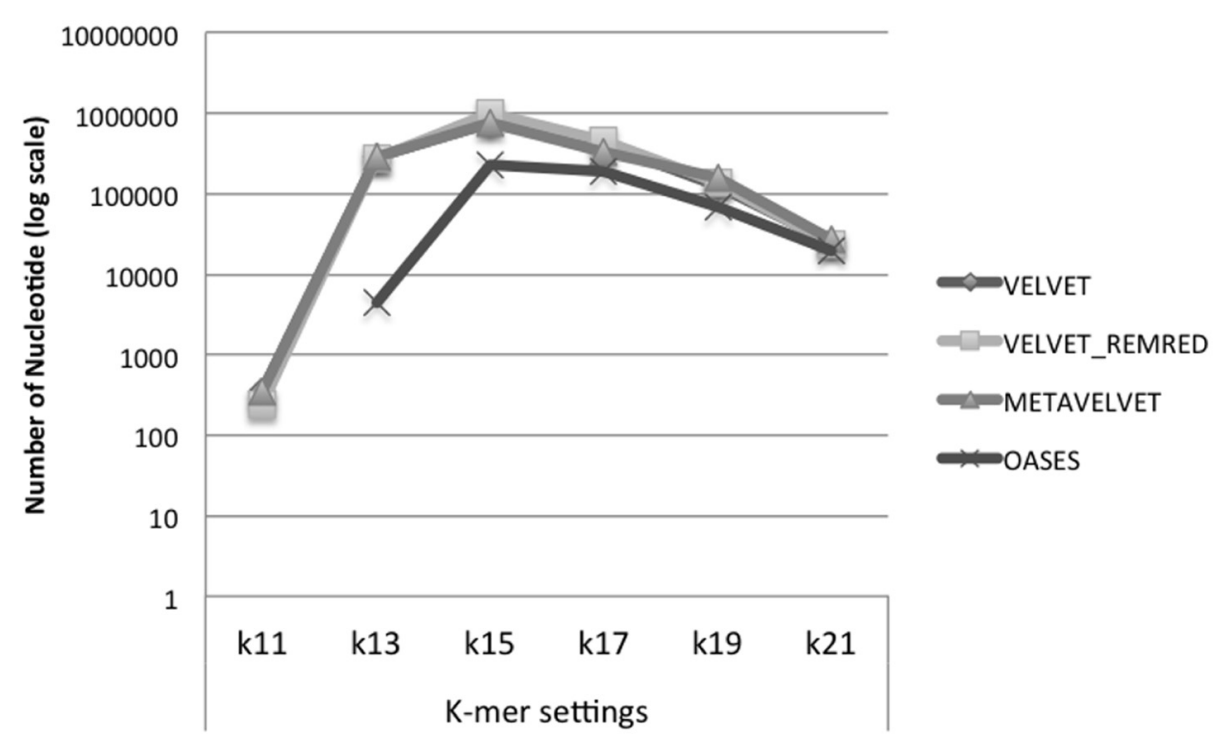

FIGURE 5 | Number of bases (log scale) in the assembled contigs obtained using Velvet, Metavelvet, and Oases short RNA assembly tools with different $\boldsymbol{k}$-mer settings. REMRED = Remove redundant.

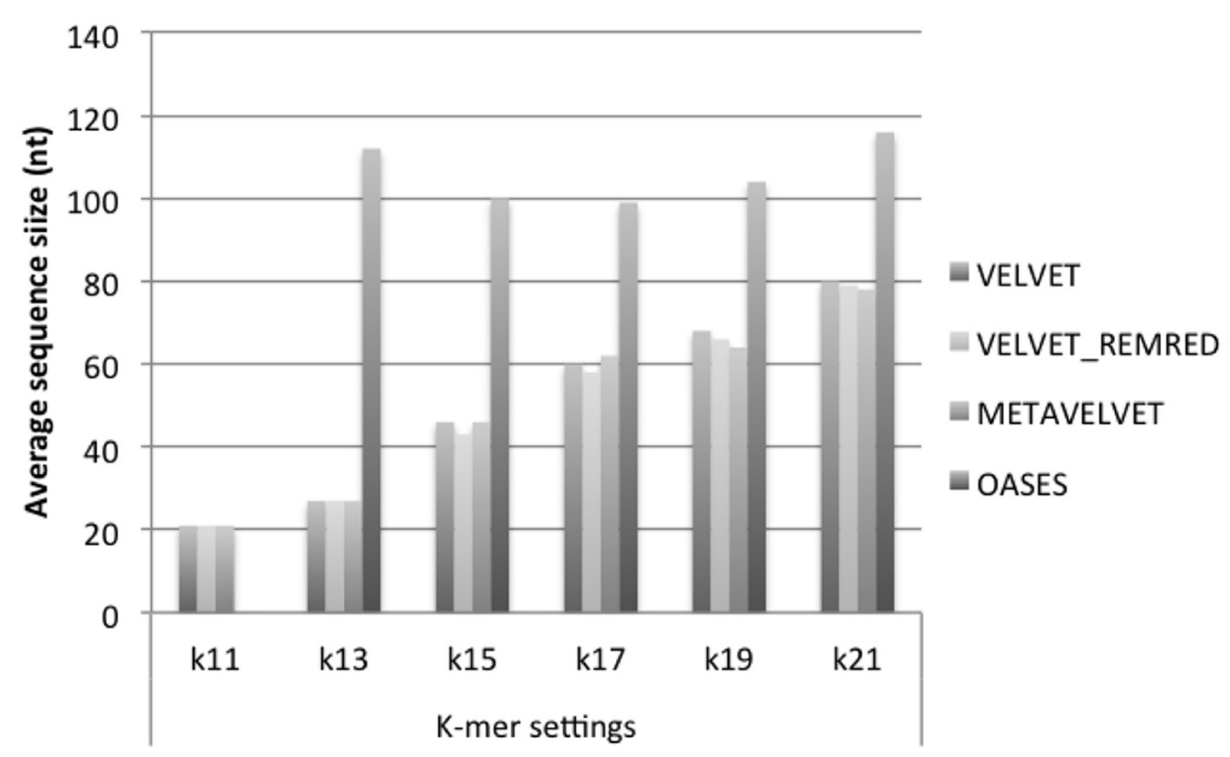

FIGURE 6 | Average size of contigs obtained using Velvet, Metavelvet, and Oases short (s)RNA assembly tools with different $k$-mer settings. REMRED = Remove redundant.

as a valid alternative, thus allowing the creation of a consensus sequence set that best represents the underlying viral population with a non-homology approach. These consensus sequences can serve as a proper basis for reference alignment and variant calling as described above. At present De Bruijn graph-based algorithms (reviewed by MacLean et al., 2009) are the methods of choice to assemble a set of NGS reads. In brief the algorithm divides the NGS reads into short sub-reads (so-called $k$-mers) and subsequently it searches the ideal assembly path in the graph through overlap between the $k$-mers. Thus, the algorithm is optimized for a fast merging of millions of short NGS reads into (large) genomic fragments. In fact, De Bruijn graph based methods such as Velvet (Zerbino and Birney, 2008) and SOAP de novo (Li et al., 2010) are widely employed for the genomic assembly of prokaryotes and eukaryotes. The algorithm, however, appears to be less suited for the assembly of fragments with unbalanced coverage distributions such as generated in RNA-Seq and metagenomic libraries. In the latter case chromosomes of different microbes are present in a metagenomics sample proportional to their relative abundance. As such the relative frequencies of short reads 


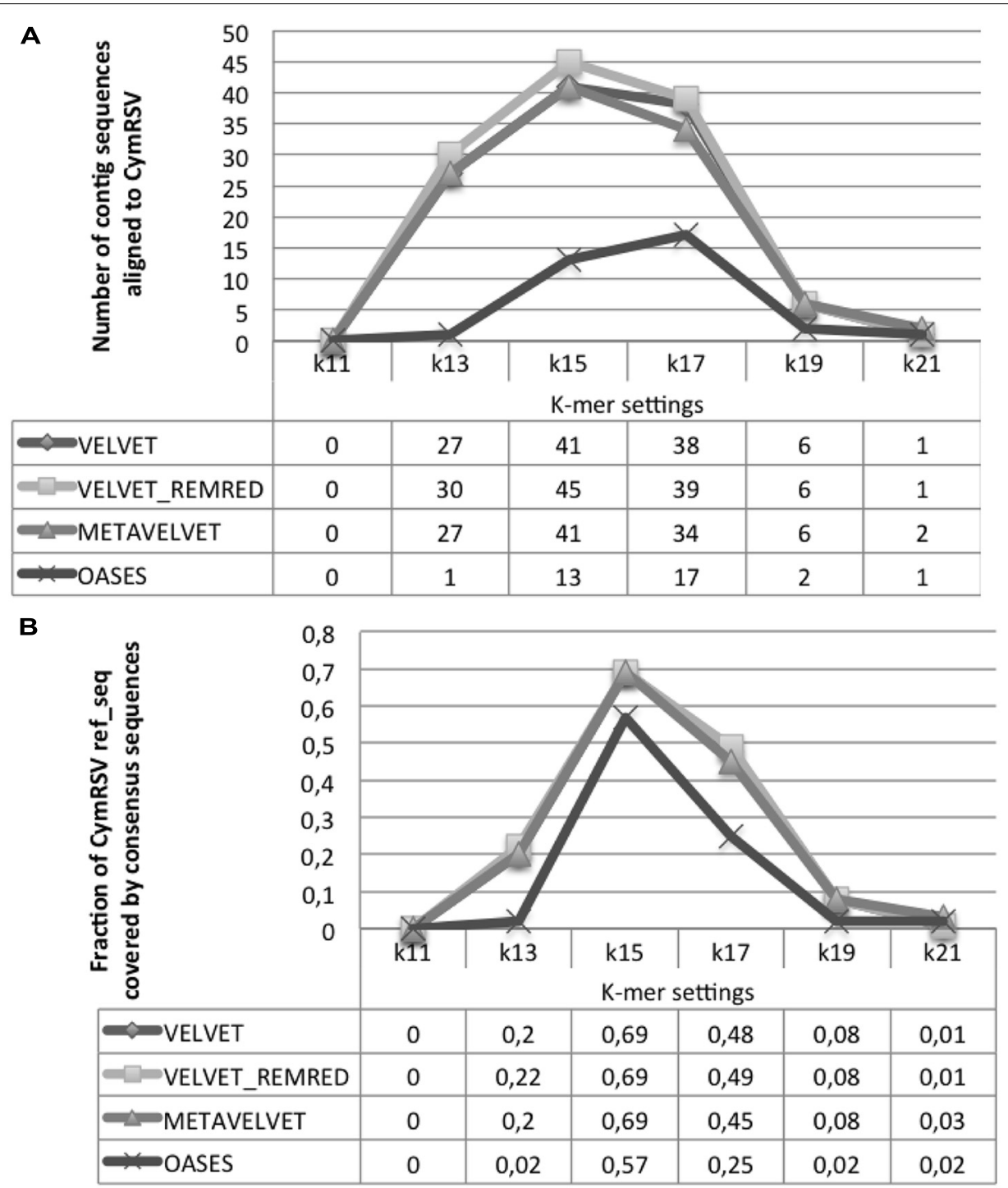

FIGURE 7 | Alignment statistics of contig sequences constructed using Velvet, Metavelvet, and Oases short RNA assembly tools with different k-mer settings. The graph and table display the number of contig sequence aligning with CymRSV ref_seq (A) and fraction of CymRSV ref_seq covered by the consensus sequence (B). REMRED = Remove redundant. covering the various nodes in the De Bruijn graph differ with respect to a standard linear genome assembly. To overcome these problems specific tools are developed for the assembly of transcriptomes, e.g., Trinity (Grabherr et al., 2011) and Oases (Schulz et al., 2012) and metagenomes [e.g., and MetaVelvet (Namiki et al., 2012) and Ray Meta (Boisvert et al., 2012)]. Potentially these methods could also be of good use for assemblies of viral metagenomes. We here apply the three well-used bioinformatics assembly tools (Velvet, Metavelvet, and Oases) and compare their relative ability to reconstruct the viral metagenome. Also we developed an inhouse modification of the standard Velvet protocol where, prior to the assembly, duplicate reads are removed (REDREM) thus taking into account issues related to unbalanced genome coverage (see Discussion). All strategies were evaluated at different $k$-mer settings.
We observe that Velvet and Metavelvet constructed the largest number of consensus sequences at all $k$-mer setting (hereafter " $k$ ") used. Surprisingly, for all tools the maximum number of consensus sequences was obtained at setting $k=15$ (Figure 4; Supplementary Data 1A). More specifically, Velvet is able to provide a higher number of consensus sequences at $k=15$ when using a nonredundant sRNA dataset, i.e., 16.604 sequences using Velvet versus 23.251 using Velvet REDREM (Supplementary Data 1A,B, respectively). Accordingly, the total number of assembled nucleotides was higher when using Velvet and Metavelvet compared to Oases (Figure 5; Supplementary Data 1).

On the other hand, when comparing the average size of the contig sequences obtained by the different methods at different $k$-mers's, Oases appears to be the best method. Indeed, except in the case of $k=11$, Oases appears to provide the longest consensus 
A

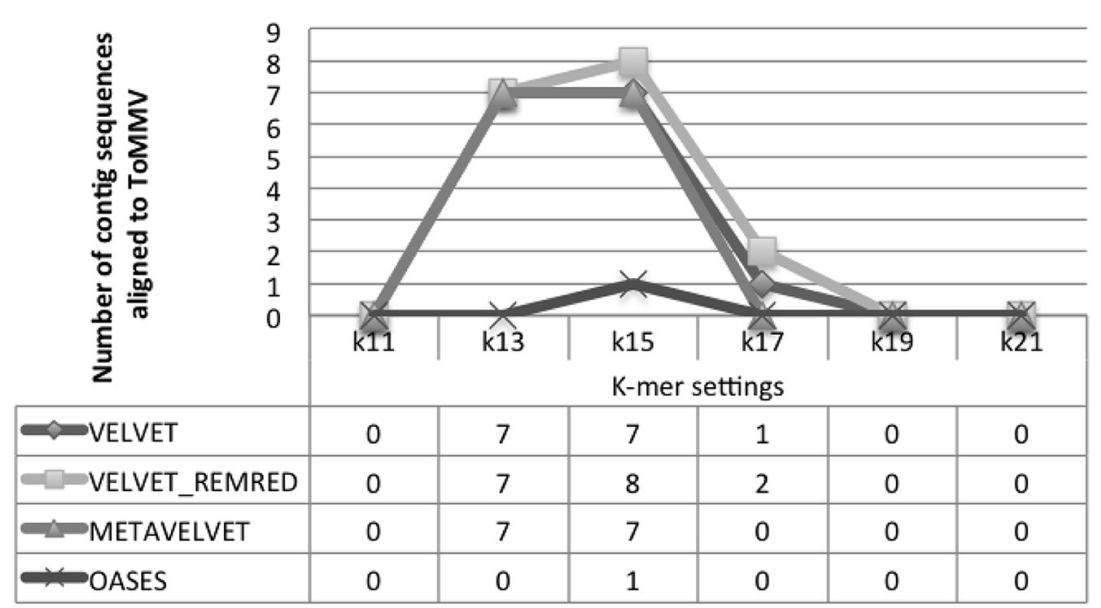

B

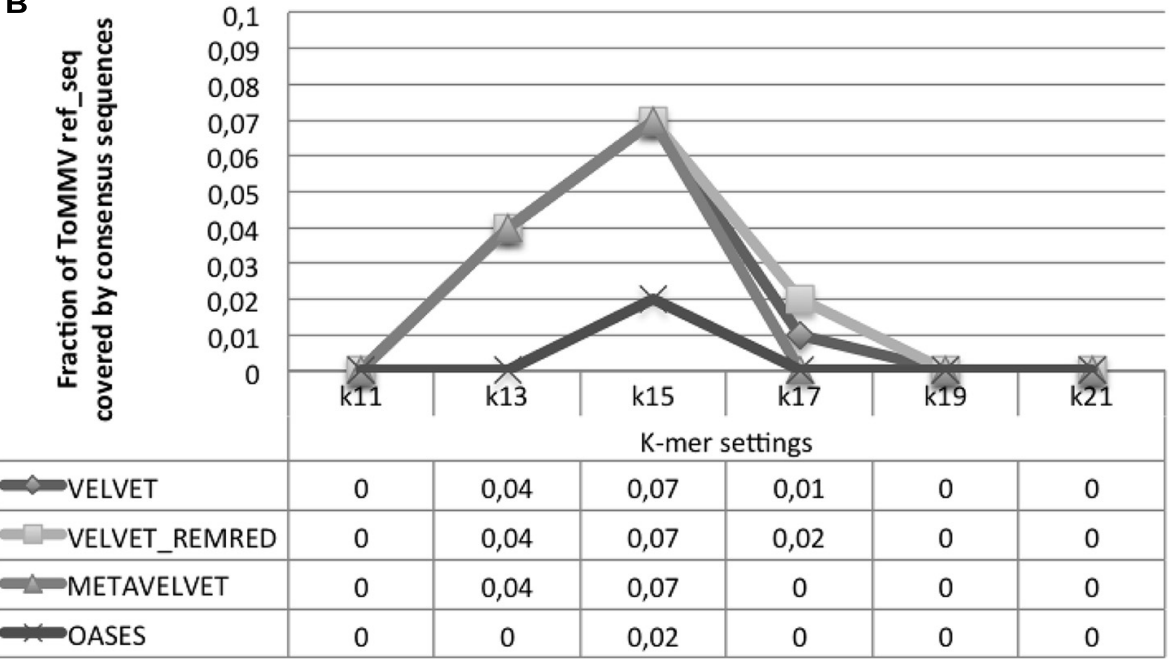

FIGURE 8 | Alignment statistics of contig sequences constructed using Velvet, Metavelvet, and Oases short RNA assembly tools with different $\boldsymbol{k}$-mer settings. The graph and table display the number of contig sequences aligning with ToMMV ref_seq (A) and the fraction of ToMMV ref_seq covered by the consensus sequence (B). REMRED = Remove redundant. sequences on average for all settings (Figure 6). Regarding the consensus sequences generated with Velvet and Metavelvet, the average length increased from $k=11$ (the lowest) to $k=21$ (the highest; Figure 6). The longest consensus sequence was obtained by Oases at $k=17$ (i.e., $919 \mathrm{nt}$ in length, Supplementary Data 1). Other tools obtained their maximum length (between 400 to $600 \mathrm{nt}$ ) at $k$-values ranging from 15 to 21 (Supplementary Data 1).

\section{DE NOVO ASSEMBLY OF CymRSV AND TOMMV}

All contig sequences obtained by different tools and settings were aligned against the CymRSV ref_seq. First Velvet REMRED and second Metavelvet and Velvet showed to be the most efficient tools by generating, respectively, 45 and 41 consensus sequences at $k=15$ (Figure 7A). For these approaches an increase or a decrease of $k$ values resulted in a sensible decrease of contig sequences aligning with the CymRSV ref_seq: e.g., in the case of Velvet REMRED the use $k=13$ or $k=17$ reduces the number of contigs to 30 and 39 , respectively, whereas for $k=15$ in total
45 contigs were assembled (Figure 7A). Moreover, when applying $k=15$ to both Velvet methods and MetaVelvet the coverage of the CymRSV genome was the highest, i.e., 0,69\% (3.247 nt out of $4.733 \mathrm{nt}$ of the CymRSV genome). Again a setting of $k=13$ or $k=17$ sensibly reduces the efficiency of the method (Figure 7B).

Oases reached a slightly lower coverage level (57\%) at $k=15$ (Figure 7B) although the average length of the 13 consensus sequences was significantly higher than the other three methods (Figure 7A).

Surprisingly, all assembly tools evaluated at $k=15$ detected a similar number of SNPs (14 in case of the Velvet methods, 12 in case of Oases; see Supplementary Data 2), which is comparable to the number of SNPs detected with the reference-guided assembly (i.e., 13, Table 1). As previously underlined, the method of $C y m R S V$ inoculation and the timing of sampling may impede a further increase of variability within the viral genome (Russo et al., 1994). 


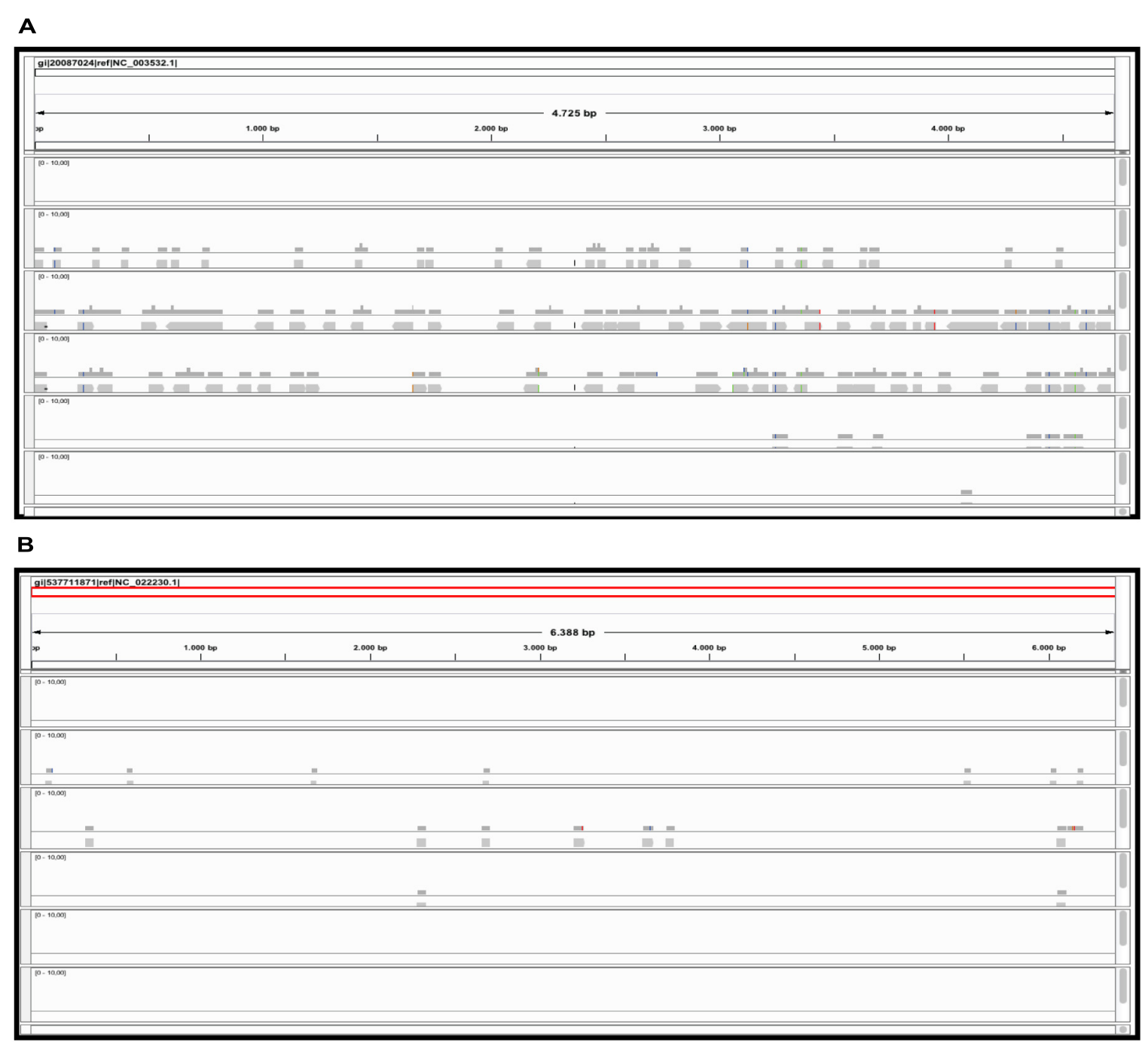

FIGURE 9 | Alignments of contig sequences obtained using Velvet REMRED, at different $\boldsymbol{k}$-mer settings. Contig distribution graph for CymRSV ref_seq (A) and ToMMV ref_seq (B) REMRED = Remove redundant.

The alignment of contigs to the ToMMV reference genome confirms the findings obtained for CymRSV; in Figure 8A we show that the $k=15$ value remains the best setting to obtain highest number of contigs and also the highest coverage (i.e., 0,07, Figure 8B). Importantly, contigs obtained at a $k$ value of 15 do not cover the exact same genome segments compared to those obtained with other $k$-mers (Figure 9B). This also holds for CymRSV analysis (Figure 9A). Thus, assemblies generated at different $k$ values may complement each other to help the reconstruction of more complete viral genomes and increase the coverage as later discussed.

Obviously, a coverage value of 0,07 cannot be considered an acceptable coverage and shows the limitations of such approach for discovering novel viral entities. In Figure 10 we compare contigs which are de novo assembled using different bioinformatics tools at a $k=15$ settings in the cases of CymRSV and ToMMV (Figures 10A,B respectively). Here we graphically confirm the findings revealed in Figure 7 and in Supplementary data 2 and 3. Indeed, the genome coverage of $T o M M V$ is significantly lower compared to that of CymRSV (Figures 7A,B) and this is at least partly due to the abundance of viral-deriving siRNAs in the library (i.e., 364.590 vs. 1.909; Tables 1 and 2).

\section{DISCUSSION}

Viral metagenomics surveys in plants have estimated that only small fractions of virus species are known. Stobbe and Roossinck (2014) have recently proposed the following classification of viruses found by metagenomics: (i) Known-known: virus species or isolates that are already known to be in the environment, (ii) Unknown-known: new virus species or isolates of a known family, or known viruses that have not been found previously in the surveyed environment, and (iii) Unknown-unknowns: viruses that are completely novel and share little to no sequence similarity with other known viruses. In this study we use 10 million sRNAs reads 


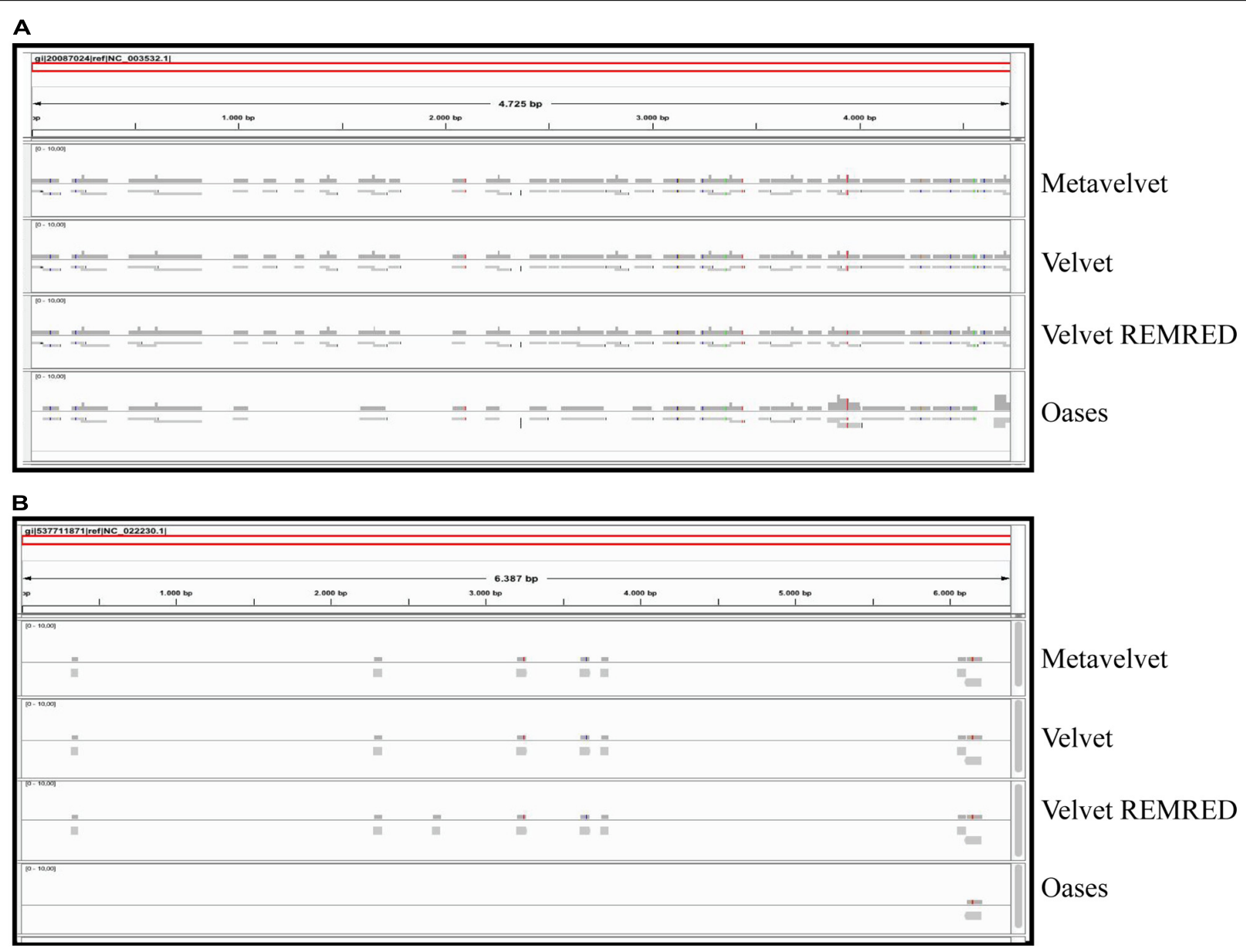

FIGURE 10 | Alignments of contig sequences obtained using Velvet, Metavelvet, and Oases short RNA assembly tools at $\boldsymbol{k}=\mathbf{1 5}$. Contig distribution graph for CymRSV ref_seq (A) and ToMMV ref_seq (B) REMRED = Remove redundant are displayed.

library (Figure 1) containing CymRSV v-siRNAs. The high coverage level of the virus is indicated by the fact that each nucleotide of the viral genomic ref_seq is represented on average 77 times in sRNAs. Subsequent variant calling showed the presence of a discrete number of SNPs (Table 1). Our data describe a typical example of metagenomics analysis of a "known-known" virus in a model-permissive system (i.e., N. benthamiana). In this case the ref_seq guided approach is able to reconstruct $99 \%$ of the genome whereas the de novo based approach is able to cover $69 \%$ of the genome. The gap between the two approaches is likely due to the heterogeneity within viral populations, the low and unbalanced genome coverage, and the rather short length of the siRNAs (around $21 \mathrm{bp}$ on average). It is expected that the assembly would at least to some extent benefit from an increased sequencing depth (i.e., by generating 20 million sRNA reads instead of 10 million) although this would of course lead to additional costs for sequencing and data managing (Seguin et al., 2014). Also it should be mentioned that the genome coverage of the de novo assembly was calculated based on a reference alignment tool using strict parameters to allow only a few mutations. It is to be expected that more permissive alignment strategies such as BLAST (Altschul et al., 1990) can detect more homologous regions between the assembly and the reference, thus allowing the reconstruction of a more complete genome. However, there is a risk that the allowance of a higher number of mismatches will contemporarily lead to the inclusion of erroneously assembled regions. Further investigations and quality assessment is needed to address this issue.

In the case of short infectious entities such as HSVd, a more permissive alignment strategy obtained by introducing more mismatches in the alignment settings (i.e., 1, 2, or 3), may better be able to cover small gaps (Figure 3). However, the approach still leaves un-resolved gaps that could be associated with specific variants in the non-conserved domain of the viroid (Keese and Symons, 1985; Visvader and Symons, 1985). This interpretation fits with the fact that up-to-date no HSVd was reported in chickpea and therefore the entity here reported could be a novel HSVd variant.

The data obtained on CymRSV indicates that for "knownknown" and some "Unknown-known" viruses the ref-seq approach may find practical (cost-effective) applications in particular for surveys of viruses for diagnosis in agro-ecosystems, plant 
population (e.g., old varieties) and single plant tissues/organs or for wider environmental studies of ecogenomics (Roossinck, 2011). Indeed, when applying the same pipeline to the sRNA library of a plant population constituting an old Chickpea variety we were able to reveal the presence of ToMMV (NC_022230), a putative novel Tobamovirus naturally infecting tomatoes in Mexico (Li et al., 2013). Note to worthy, we show through a metagenomics approach that the ToMMV is already present in Europe and that in can be hosted by $C$. arietinum. The ToMMV has been just proposed as a novel species of the Tobamovirus genus based on sequence similarity with other species of the genus. Phylogenetic analysis shows that ToMMV was clustered together with a group of Tobamoviruses mainly infecting solanaceous plants and therefore the presence in chickpea may give good reasons for further characterization of the viral genome, i.e., by generating a higher sRNA sequencing depth with the aim to increase the overall coverage. In cases where plant populations are studied classical molecular approaches are not always applicable. Indeed, an RT-PCR strategy was designed on assembled contigs and attempted on total RNA (see Materials and Methods) but no amplified products were observed. This may be due to the very low titer of virus in the tissues and/or to the infection of a discrete number of plants within the population composing the variety. The presence of a Tobamovirus into old varieties of Chickpea in Puglia is not surprising since this genus of plant viruses is known to be hosted by a wide range of plant species, including legumes. Moreover, all viral species are known to be transmitted mechanically and also through contaminations of the seed teguments (Broadbent, 1965).

In summary, a reference-guided approach appeared the most efficient in reconstructing viral metagenomes. Our results indicate that, using an appropriate short-read alignment mapping tool, even low abundant viruses can be well reconstructed. (e.g., at average sequencing depth lower than $7 \%$, still $87 \%$ of the virus genome could be assembled). The de novo assembly based approaches reached a non-appreciable genome coverage and show a relatively high degree of fragmentation. Nonetheless the contigs generated were sufficiently long for assigning a proper taxonomic classification. Remarkably, the removal of duplicate sequences or the use of Metavelvet assembly software, which is specifically designed for metagenomics applications, did not contribute to more complete assemblies (i.e., longer contigs). At different $k$-mer settings all genome-based assembly strategies used yield similar genome coverage. In contrast, the use of a transcriptomebased method such as Oases resulted in longer contigs and may therefore be the method of choice for v-siRNA-based assemblies. Even if the total genome coverage was lower than in the case of genome-based assembly strategies, the increased average length may provide better anchor points for primer design of PCR products.

Thus, we deduce that transcriptome-based algorithms (i.e., those having adapted the original De Bruijn graph to assemble differentially expressed non-repetitive genes) could better manage the (large) differences between frequencies of sRNA reads covering the various viruses. Strategies based on the original De Bruijn graph algorithm instead attempt to remove $k$-mers with extreme abundance (also simulated by our REMRED approach).
Moreover metagenomic-based assembly strategies need to overcome genome-repetitiveness in addition to differences in genome coverage: it may be that the complexity of these issues is higher than the v-siRNA assembly problem where genome repetitiveness is less of an issue.

We conclude that no method or particular $k$-mer setting was able to generate a full coverage, but also that different parameter settings led to assembly of unique (non-overlapping) v-siRNAs. Thus the use of a consensus-based strategy, where a master consensus genome is constructed from multiple assemblies (i.e., at different settings) could potentially be a more robust approach to reconstruct more complete viral genomes.

\section{ACKNOWLEDGMENTS}

The work was mainly supported by the project SaVeGrainINPuglia - Progetti integrati per la biodiversità “PSR” Regione Puglia FEASR 2007-2013. Reg. (CE) 1698/2005.

\section{SUPPLEMENTARY MATERIAL}

The Supplementary Material for this article can be found online at: http://www.frontiersin.org/journal/10.3389/fmicb.2014.00790/ abstract

\section{REFERENCES}

Aliyari, R., and Ding, S. W. (2009). RNA-based viral immunity initiated by the Dicer family of host immune receptors. Immunol. Rev. 227, 176-188. doi: 10.1111/j.1600-065X.2008.00722.x

Altschul, S. F., Gish, W., Miller, W., Myers, E. W., and Lipman, D. J. (1990). Basic local alignment search tool. J. Mol. Biol. 215, 403-410. doi: 10.1016/S00222836(05)80360-2

Barba, M., Czosnek, H., and Hadidi, A. (2014). Historical perspective, development and applications of next-generation sequencing in plant virology. Viruses 6, 106136. doi: 10.3390/v6010106

Boisvert, S., Raymond, F., Godzaridis, E., Laviolette, F., and Corbeil, J. (2012). Ray Meta: scalable de novo metagenome assembly and profiling. Genome Biol. 13:R122. doi: 10.1186/gb-2012-13-12-r122

Broadbent, L. (1965). The epidemiology of TMV. Ann. Appl. Biol. 56, 177-205. doi: 10.1111/j.1744-7348.1965.tb01227.x

Candresse, T., Filloux, D., Muhire, B., Julian, C., Galzi, S., Fort, G., et al. (2014). Appearances can be deceptive: revealing a hidden viral infection with deep sequencing in a plant quarantine context. PLOS ONE 9:e102945. doi: 10.1371/journal.pone.0102945

De Souza, J., Fuentes, S., Savenkov, E. I., Cuellar, W., and Kreuze, J. F. (2013). The complete nucleotide sequence of sweet potato C6 virus: a carlavirus lacking a cysteine-rich protein. Arch. Virol. 158, 1393-1396. doi: 10.1007/s00705-0131614-x

Ding, S. W., and Voinnet, O. (2007). Antiviral immunity directed by small RNAs. Cell 130, 413-426. doi: 10.1016/j.cell.2007.07.039

Domingo, E., Sheldon, J., and Perales, C. (2012). Viral quasispecies evolution. Microbiol. Mol. Biol. Rev. 76, 159-216. doi: 10.1128/MMBR.05023-11

Donaire, L., Barajas, D., Martinez-Garcia, B., Martinez-Priego, L., Pagan, I., and Llave, C. (2008). Structural and genetic requirements for the biogenesis of tobacco rattle virus-derived small interfering RNAs. J. Virol. 82, 5167-5177. doi: 10.1128/JVI.00272-08

Garcia, D., Garcia, S., Pontier, D., Marchais, A., Renou, J. P., Lagrange, T., et al. (2012). Ago hook and RNA helicase motifs underpin dual roles for SDE3 in antiviral defense and silencing of nonconserved intergenic regions. Mol. Cell 48, 109-120. doi: 10.1016/j.molcel.2012.07.028

Garcia-Ruiz, H., Takeda, A., Chapman, E. J., Sullivan, C. M., Fahlgren, N., Brempelis, K. J., et al. (2010). Arabidopsis RNA-dependent RNA polymerases and dicer-like proteins in antiviral defense and small interfering RNA biogenesis during Turnip Mosaic Virus infection. Plant Cell 22, 481-496. doi: 10.1105/tpc.109.073056

Giampetruzzi, A., Roumi, V., Roberto, R., Malossini, U., Yoshikawa, N., La Notte, P., et al. (2012). A new grapevine virus discovered by deep sequencing of virus- 
and viroid-derived small RNAs in Cv Pinot gris. Virus Res. 163, 262-268. doi: 10.1016/j.virusres.2011.10.010

Grabherr, M. G., Haas, B. J., Yassour, M., Levin, J. Z., Thompson, D. A., Amit, I., et al. (2011). Full-length transcriptome assembly from RNA-Seq data without a reference genome. Nat. Biotechnol. 29, 644-652. doi: 10.1038/nbt.1883

Huson, D. H., Mitra, S., Ruscheweyh, H. J., Weber, N., and Schuster, S. C. (2011). Integrative analysis of environmental sequences using MEGAN4. Genome Res. 21, 1552-1560. doi: 10.1101/gr.120618.111

Johansen, L. K., and Carrington, J. C. (2001). Silencing on the spot. Induction and suppression of RNA silencing in the Agrobacterium-mediated transient expression system. Plant Physiol. 126, 930-938. doi: 10.1104/pp.126.3.930

Keese, P., and Symons, R. H. (1985). Domains in viroids: evidence of intermolecular RNA rearrangements and their contribution to viroid evolution. Proc. Natl. Acad Sci. U.S.A. 82, 4582-4586. doi: 10.1073/pnas.82.14.4582

Kreuze, J., Koenig, R., De Souza, J., Vetten, H. J., Muller, G., Flores, B., et al. (2013). The complete genome sequences of a Peruvian and a Colombian isolate of Andean potato latent virus and partial sequences of further isolates suggest the existence of two distinct potato-infecting tymovirus species. Virus Res. 173, 431-435. doi: 10.1016/j.virusres.2013.01.014

Kreuze, J. F., Perez, A., Untiveros, M., Quispe, D., Fuentes, S., Barker, I., et al. (2009). Complete viral genome sequence and discovery of novel viruses by deep sequencing of small RNAs: a generic method for diagnosis, discovery and sequencing of viruses. Virology 388, 1-7. doi: 10.1016/j.virol.2009. 03.024

Kurtz, S., Phillippy, A., Delcher, A. L., Smoot, M., Shumway, M., Antonescu, C., et al. (2004). Versatile and open software for comparing large genomes. Genome Biol. 5, R12. doi: 10.1186/gb-2004-5-2-r12

Li, H., and Durbin, R. (2009). Fast and accurate short read alignment with Burrows-Wheeler transform. Bioinformatics 25, 1754-1760. doi: 10.1093/bioinformatics/btp324

Li, H., Handsaker, B., Wysoker, A., Fennell, T., Ruan, J., Homer, N., et al. (2009). The Sequence Alignment/Map format and SAMtools. Bioinformatics 25, 2078-2079. doi: 10.1093/bioinformatics/btp352

Li, R., Fan, W., Tian, G., Zhu, H., He, L., Cai, J., et al. (2010). The sequence and de novo assembly of the giant panda genome. Nature 463, 311-317. doi: 10.1038 /nature08696

Li, R., Gao, S., Fei, Z., and Ling, K. S. (2013). Complete genome sequence of a new Tobamovirus naturally infecting tomatoes in Mexico. Genome Announc. 1:e00794-13.

Loconsole, G., Saldarelli, P., Doddapaneni, H., Savino, V., Martelli, G. P., and Saponari, M. (2012). Identification of a single-stranded DNA virus associated with citrus chlorotic dwarf disease, a new member in the family Geminiviridae. Virology 432, 162-172. doi: 10.1016/j.virol.2012. 06.005

MacLean, D., Jones, J. D., and Studholme, D. J. (2009). Application of 'nextgeneration' sequencing technologies to microbial genetics. Nat. Rev. Microbiol. 7 , 287-296.

Marais, A., Faure, C., Mustafayev, E., Barone, M., Alioto, D., and Candresse, T. (2015). Characterization by deep sequencing of Prunus virus T, a novel Tepovirus infecting Prunus species. Phytopathology 105, 135-140. doi: 10.1094/PHYTO-0414-0125-R

Massart, S., Olmos, A., Jijakli, H., and Candresse, T. (2014). Current impact and future directions of high throughput sequencing in plant virus diagnostics. Virus Res. 188, 90-96. doi: 10.1016/j.virusres.2014.03.029

Mokili, J. L., Rohwer, F., and Dutilh, B. E. (2012). Metagenomics and future perspectives in virus discovery. Curr. Opin. Virol. 2, 63-77. doi: 10.1016/j.coviro.2011.12.004

Moxon, S., Jing, R., Szittya, G., Schwach, F., Rusholme Pilcher, R. L., Moulton, V., et al. (2008). Deep sequencing of tomato short RNAs identifies microRNAs targeting genes involved in fruit ripening. Genome Res. 18, 1602-1609. doi: 10.1101/gr.080127.108

Namiki, T., Hachiya, T., Tanaka, H., and Sakakibara, Y. (2012). MetaVelvet: an extension of Velvet assembler to de novo metagenome assembly from short sequence reads. Nucleic Acids Res. 40:e155. doi: 10.1093/nar/ gks678

Navarro, B., Pantaleo, V., Gisel, A., Moxon, S., Dalmay, T., Bisztray, G., et al. (2009). Deep sequencing of viroid-derived small RNAs from grapevine provides new insights on the role of RNA silencing in plant-viroid interaction. PLOS ONE 4:e7686. doi: 10.1371/journal.pone.00 07686

Pantaleo, V., and Burgyan, J. (2008). Cymbidium ringspot virus harnesses RNA silencing to control the accumulation of virus parasite satellite RNA. J. Virol. 82, 11851-11858. doi: 10.1128/JVI.01343-08

Pantaleo, V., Saldarelli, P., Miozzi, L., Giampetruzzi, A., Gisel, A., Moxon, S., et al. (2010). Deep sequencing analysis of viral short RNAs from an infected Pinot Noir grapevine. Virology 408, 49-56. doi: 10.1016/j.virol.2010.09.001

Pantaleo, V., Szittya, G., and Burgyan, J. (2007). Molecular bases of viral RNA targeting by viral small interfering RNA-programmed RISC. J. Virol. 81, 37973806. doi: 10.1128/JVI.02383-06

Rajagopalan, R., Vaucheret, H., Trejo, J., and Bartel, D. P. (2006). A diverse and evolutionarily fluid set of microRNAs in Arabidopsis thaliana. Genes Dev. 20, 3407-3425. doi: 10.1101/gad.1476406

Robinson, J. T., Thorvaldsdottir, H., Winckler, W., Guttman, M., Lander, E. S., Getz, G., et al. (2011). Integrative genomics viewer. Nat. Biotechnol. 29, 24-26. doi: $10.1038 /$ nbt. 1754

Roossinck, M. J. (2011). Environmental viruses from biodiversity to ecology. Curr. Opin. Virol. 1, 50-51. doi: 10.1016/j.coviro.2011.05.012

Roossinck, M. J. (2012). Plant virus metagenomics: biodiversity and ecology. Annu. Rev. Genet. 46, 359-369. doi: 10.1146/annurev-genet-110711-155600

Ruiz-Ferrer, V., and Voinnet, O. (2009). Roles of plant small RNAs in biotic stress responses. Annu. Rev. Plant Biol. 60, 485-510. doi: 10.1146/annurev.arplant.043008.092111

Russo, M., Burgyan, J., and Martelli, G. P. (1994). Molecular biology of tombusviridae. Adv. Virus Res. 44, 381-428. doi: 10.1016/S0065-3527(08)60334-6 Schloss, L., Falk, K. I., Skoog, E., Brytting, M., Linde, A., and Aurelius, E. (2009). Monitoring of Herpes Simplex Virus DNA types 1 and 2 viral load in cerebrospinal fluid by real-time PCR in patients with herpes simplex encephalitis. J. Med. Virol. 81, 1432-1437. doi: 10.1002/jmv.21563

Schulz, M. H., Zerbino, D. R., Vingron, M., and Birney, E. (2012). Oases: robust de novo RNA-seq assembly across the dynamic range of expression levels. Bioinformatics 28, 1086-1092. doi: 10.1093/bioinformatics/bts094

Seguin, J., Rajeswaran, R., Malpica-Lopez, N., Martin, R. R., Kasschau, K., Dolja, V. V., etal. (2014). De novo reconstruction of consensus master genomes of plant RNA and DNA viruses from siRNAs. PLoS ONE 9:e88513. doi: 10.1371/journal.pone.0088513

Shimura, H., and Pantaleo, V. (2011). Viral induction and suppression of RNA silencing in plants. Biochim. Biophys. Acta 1809, 601-612. doi: 10.1016/j.bbagrm.2011.04.005

Sorefan, K., Pais, H., Hall, A. E., Kozomara, A., Griffiths-Jones, S., Moulton, V., et al. (2012). Reducing ligation bias of small RNAs in libraries for next generation sequencing. Silence 3:4. doi: 10.1186/1758-907X-3-4

Stobbe, A. H., and Roossinck, M. J. (2014). Plant virus metagenomics: what we know and why we need to know more. Front. Plant Sci. 5:150. doi: 10.3389/fpls.2014.00150

Szittya, G., Moxon, S., Pantaleo, V., Toth, G., Rusholme Pilcher, R. L., Moulton, V., et al. (2010). Structural and functional analysis of viral siRNAs. PLoS Pathog. 6:e1000838. doi: 10.1371/journal.ppat.1000838

Vaistij, F. E., and Jones, L. (2009). Compromised virus-induced gene silencing in RDR6-deficient plants. Plant Physiol. 149, 1399-1407. doi: 10.1104/pp.108.132688

Visvader, J. E., and Symons, R. H. (1985). Eleven new sequence variants of citrus exocortis viroid and the correlation of sequence with pathogenicity. Nucleic Acids Res. 13, 2907-2920. doi: 10.1093/nar/13.8.2907

Wang, X. B., Wu, Q., Ito, T., Cillo, F., Li, W. X., Chen, X., et al. (2010). RNAi-mediated viral immunity requires amplification of virus-derived siRNAs in Arabidopsis thaliana. Proc. Natl. Acad. Sci. U.S.A. 107, 484-489. doi: 10.1073/pnas.0904086107

Wassenegger, M., and Krczal, G. (2006). Nomenclature and functions of RNA-directed RNA polymerases. Trends Plant Sci. 11, 142-151. doi: 10.1016/j.tplants.2006.01.003

Wu, Q., Luo, Y., Lu, R., Lau, N., Lai, E. C., Li, W. X., et al. (2010). Virus discovery by deep sequencing and assembly of virus-derived small silencing RNAs. Proc. Natl. Acad. Sci. U.S.A. 107, 1606-1611. doi: 10.1073/pnas.09113 53107

Wu, Q., Wang, Y., Cao, M., Pantaleo, V., Burgyan, J., Li, W. X., et al. (2012). Homology-independent discovery of replicating pathogenic circular RNAs by 
deep sequencing and a new computational algorithm. Proc. Natl. Acad. Sci. U.S.A. 109, 3938-3943. doi: 10.1073/pnas.1117815109

Zerbino, D. R., and Birney, E. (2008). Velvet: algorithms for de novo short read assembly using de Bruijn graphs. Genome Res. 18, 821-829. doi: 10.1101/gr.074492.107

Conflict of Interest Statement: The authors declare that the research was conducted in the absence of any commercial or financial relationships that could be construed as a potential conflict of interest.

Received: 06 November 2014; paper pending published: 25 November 2014; accepted: 22 December 2014; published online: 27 January 2015.
Citation: Pirovano W, Miozzi L, Boetzer M and Pantaleo V (2015) Bioinformatics approaches for viral metagenomics in plants using short RNAs: model case of study and application to a Cicer arietinum population. Front. Microbiol. 5:790. doi: 10.3389/fmicb.2014.00790

This article was submitted to Virology, a section of the journal Frontiers in Microbiology. Copyright (c) 2015 Pirovano, Miozzi, Boetzer and Pantaleo. This is an open-access article distributed under the terms of the Creative Commons Attribution License (CC BY). The use, distribution or reproduction in other forums is permitted, provided the original author(s) or licensor are credited and that the original publication in this journal is cited, in accordance with accepted academic practice. No use, distribution or reproduction is permitted which does not comply with these terms. 